do they report whether treatment with AEF or SN alone leads to the induction of RAGE. In this mouse model, hepatic amyloid accumulates after 48-72 hours ${ }^{7}$, and monocyte/macrophages have not been associated with these early liver deposits in mice or humans. Additional in vivo experiments examining different cell types associated with AA amyloid deposits in different organs may help clarify the relationship of monocyte/macrophage RAGE expression and AA amyloid deposition.

Yan et al. also made the tantalizing observation that RAGE blockade through the administration of soluble (s)RAGE or treatment with an antibody fragment against RAGE prevents NF- $\mathrm{kB}$ activation, induction of the cell stress response and AA deposition in this mouse model ${ }^{6}$. However, further work will be required to determine the mechanism of action and the possible plieotropic effects of sRAGE on monocyte/macrophage. The antibody against RAGE and sRAGE may be exerting their AA-inhibitory proper- ties through a mechanism that does not involve interference with the binding of RAGE to SAA or AA amyloid fibrils.

Although additional studies are needed to support the conclusions proposed in this study, the findings are in keeping with clinical observations and do indicate a specific mechanism by which at least one form of amyloid (AA) may influence cellular (monocyte/macrophage) function. The data also demonstrate that amyloid is no 'innocent bystander'. The observation that RAGE has a propensity to interact with various forms of amyloid fibrils indicates the possibility of new therapeutic approaches to amyloidosis. Further studies will demonstrate whether these concepts can be extended to other forms of amyloid accumulations adjacent to other cell types and that may involve additional cell receptors.

1. Westermark, P. Classification of amyloid fibril proteins and their precursors: an ongoing discussion. Amyloid 4, 216-218 (1997).

2. Haggqvist, B. et al. Medin: An integral fragment of aortic smooth muscle cell-produced lactadherin forms the most common human amyloid. Proc. Natl. Acad. Sci. USA 96, 8669-8674 (1999).

3. Kisilevsky, R. \& Fraser, P. E. Ab amyloidogenesis: unique or variation on a systemic theme? Crit. Rev. Biochem. Molec. Biol. 32, 361-404 (1997).

4. Lorenzo, A. \& Yankner, B. A. Beta-amyloid neurotoxicity requires fibril formation and is inhibited by Congo red. Proc. Natl. Acad. Sci. USA 91, 12243-12247 (1994).

5. Janciauskiene, S. \& Ahren, B. Different sensitivity to the cytotoxic action of IAPP fibrils in two insulin-producing cell lines, HIT-T15 and RINm5F cells. Biochem. Biophys. Res. Commun. 251, 888-893 (1998).

6. Yan, S.D. et al. Receptor-dependent cell stress and amyloid accumulation in systemic amyloidosis. Nature Med. 6, 643-651 (2000).

7. Kisilevsky, R. \& Boudreau, L. The kinetics of amyloid deposition: I. The effect of amyloid enhancing factor and splenectomy. Lab. Invest. 48, 53-59 (1983).

8. Snow, A. D., Kisilevsky, R., Stephens, C., \& Anastassiades, T. Characterization of tissue and plasma glycosaminoglycans during experimental AA amyloidosis and acute inflammation. Quantitative and qualitative analysis. Lab. Invest. 56, 665-675 (1987).

Department of Pathology, Queen's University and The Syl and Molly Apps Research Center Kingston General Hospital

Kingston, Ontario Canada

K7L $3 N 6$

Email: kisilevsky@cliff.path.queensu.ca

\title{
Is neural cell transplantation readv for the clinic?
}

The human brain has little capacity for self-repair, and developing therapies for brain damage caused by disease or injury remains one of the greatest clinical challenges. There has been much interest in the possibility of treating brain damage by transplanting new cells, but this radical approach is still at an early experimental stage. In a commentary in the June issue of Nature Neuroscience, two leading researchers in the field of neural transplantation, Anders Björklund and Olle Lindvall of Lund University in Sweden, discuss some of the scientific and ethical challenges facing this emerging field.

Not all forms of brain damage are equal, say the authors. They discuss four conditions for which clinical trials are already underway in various centers around the world: Parkinson disease, Huntington disease, epilepsy and stroke. In the first two examples, the authors argue, there are good reasons to move ahead with clinical trials. Encouraging results have been obtained with good animal models, and there is a clear understanding of why the procedure works and how it might be improved. For Parkinson disease, there are also some preliminary data to indicate that grafts may be beneficial in human patients. Grafted neurons can survive for long periods of time in the human brain, despite the progression of the disease in the surrounding tissue. (The picture shows the distribution of ${ }^{18} \mathrm{~F}$-dopa, a marker of dopaminergic terminals, in a patient who received a unilateral transplant of dopamine neurons into the putamen 10 years earlier.) More importantly, the grafts retain the ability to function properly in their new environment, and this can be measured in living patients and correlated with improvements in their clinical symptoms.
In the cases of epilepsy and stroke, however, Björklund and Lindvall are more critical. Although some animal studies have shown improvement of behavioral symptoms after neural transplantation, the reasons for these effects are unclear. Without a better understanding of the underlying science, say the authors, clinical trials for these disorders are still premature. They acknowledge, however, that the decision to proceed with clinical trials involves striking a difficult balance between the scientific uncertainties, the possible risks to the patients, the desperation of those for whom no alternative treatments are available, and the commercial interests of the companies sponsoring the trials. The authors emphasize the need for a cautious approach, and warn against the danger (demonstrated by recent events in the field of gene therapy) of promising too much, too soon.

Finally, the authors discuss the need for better sources of cells for transplantation. Although a variety of cell types have been transplanted into the brains of human patients, ranging from fetal pig neurons to cells derived from a human teratocarcinoma, the most promising results so far have been obtained with cells from aborted human fetuses. This raises both practical and ethical concerns, and the authors argue that transplantation therapy is likely to remain highly experimental unless the field can develop alternative sources that are more readily available and less controversial.

Charles Jennings Editor, Nature Neuroscience 